\title{
Analysis of protein folding equilibria by nano-electrospray-ionization mass spectrometry
}

\author{
Irena Matecko, Norbert Müller and Rita Grandori * \\ Institute of Chemistry, Johannes Kepler University, Altenbergerstrasse 69, A-4040 Linz, Austria
}

\begin{abstract}
This paper summarizes recent findings from our group concerning applications of electrospray-ionization mass spectrometry (ESI-MS) to the study of protein folding. Nano-ESI-MS was employed for the investigation of protein conformational states under varying solvent conditions and at varying values of the instrumental parameters. The effect of trifluoroethanol (TFE) on a peptide and acid-unfolded cytochrome $c$ (cyt $c$ ), monitored by circular dichroism (CD) and time-of-flight ESI-MS, illustrates the specificity of the latter technique for features of protein tertiary structure. Measurements on marginally stable protein states indicate that it is possible to identify operational conditions for nano-ESI-MS in which none of the instrumental parameters limits conformational stability in the protein sample. Results described here show that changes in the charge-state distributions (CSDs) under controlled conditions allow not only discriminating between native and denatured states, but also monitoring minor conformational changes, like the transition from molten globule to native state. These studies underscore the potential of mass spectrometry methods for the analysis of heterogeneous samples and, in particular, for the characterization of dynamic equilibria involving different conformational states.
\end{abstract}

\section{Introduction}

Mass spectrometry (MS) can be applied to the study of protein folding in two conceptually different ways. One approach is to use high-accuracy mass determination by MS in order to monitor conformationdependent reactions, such as alkylation [1] and hydrogen/deuterium exchange [2]. An alternative strategy is to directly exploit the conformation dependence of protein mass spectra obtained by electrosprayionization (ESI) [3]. The ESI-MS technique allows the analysis of multiply charged, gaseous, protein ions starting from liquid probes [4]. The observed CSDs are affected by the conformation of the protein in solution, before its transfer to the gas phase. Compact conformations typically result in lower charge states (higher $\mathrm{m} / \mathrm{z}$ values) than unfolded conformations of the same molecule [5]. High sensitivity and high throughput are appealing features of this method to probe protein conformational states in solution. Nevertheless, not always good reproducibility of the results and disagreements about the mechanism underlying the conformation dependence of CSDs has led to skepticism about a general applicability of this approach $[6,7]$.

A recent report suggests an interpretation of the electrospray process in protein solutions that brings previously unexplained results into a consistent picture [8]. In this view, intramolecular interactions involving ionizable side chains in folded protein molecules cause substantial preservation of the preexisting charge states. Loss of native protein structure leads to increased reactivity towards ionization and neutralization reactions, resulting in higher net charges of the detected ions. This analysis corroborates and extends the original hypothesis formulated by Katta and Chait [9], and supports confident

\footnotetext{
*Corresponding author. Tel.: +43 732 24688763; Fax: +43 732 24688747; E-mail: rita.grandori@jku.at.
} 
interpretation of structural effects on protein ESI-MS spectra. The present contribution summarizes experiments that have been recently carried out in our laboratory analyzing protein conformational transitions in solution by ESI-MS. The results indicate that the application of this technique under highly controlled experimental conditions provides reliable information about the composition of structurally heterogeneous samples.

\section{Results}

\subsection{Specificity for protein tertiary structure}

Several reports have presented evidence for the independence of ESI-MS spectra of protein secondary structure [10-13]. Protein conformational states known to differ in secondary structure content gave rise to similar CSDs. On the other hand, conformational changes affecting tertiary structure only, could be readily detected by ESI-MS. Stabilization of protein secondary structure by TFE has been extensively employed for folding studies [14,15]. We investigated the effect of TFE on ESI-MS spectra of a short peptide (H-peptide) and on acid-denatured cyt $c$ [13]. In both cases, the addition of TFE in the concentration range 2.5-20\% causes pronounced coil-to-helix transitions, as documented by far-UV CD spectroscopy. Nevertheless, the corresponding ESI-MS spectra show very limited changes. Figure 1 shows the CD spectra of $5 \mu \mathrm{M}$ cyt $c$ at $\mathrm{pH} 2.2$ in the presence of increasing TFE concentrations. The progressive coilto-helix transition is characterized by an isodichroic point at $203 \mathrm{~nm}$. The spectrum at $20 \%$ TFE shows the minima at 208 and $222 \mathrm{~nm}$ and the maximum at $192 \mathrm{~nm}$, typical of structures with high $\alpha$-helix content.

The same solvent conditions employed for CD experiments were investigated by nano-ESI-MS $[16,17]$. This technique is compatible with mild temperature and voltage conditions and is, therefore, well suited for the analysis of marginally stable protein structures. Moreover, the CSDs obtained by nano-ESI-MS are particularly narrow, improving the resolution of distinct envelops characterizing heterogeneous samples. Figure 2 displays nano-ESI-MS spectra of $5 \mathrm{mM}$ cyt $c$ at $\mathrm{pH} 2.2$ in the presence of $0 \%$ and 20\% TFE. These spectra are very similar. This result indicates that the $\alpha$-helix content of protein or peptide samples does not directly affect ESI-MS spectra. This conclusion is also supported by

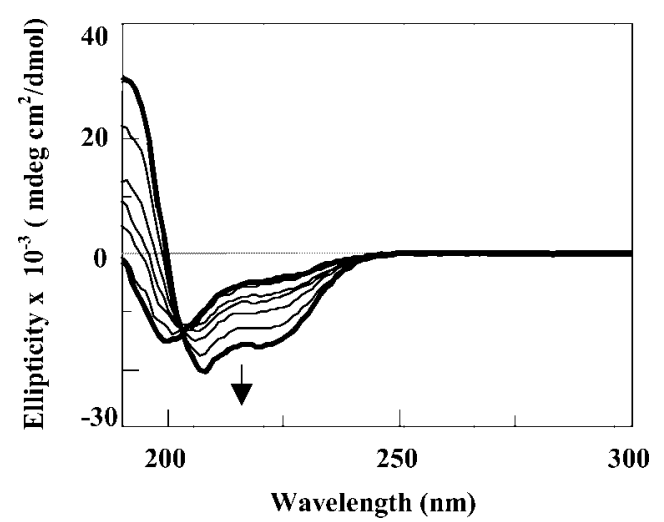

Fig. 1. CD spectra of $80 \mu \mathrm{M}$ cyt $c$ in water-acetate $\mathrm{pH} 2.2$ at increasing TFE concentrations. The arrow indicates the direction of the changes induced by increasing TFE concentrations, which are $0 \%, 2.5 \%, 5 \%, 7.5 \%, 10 \%, 15 \%$ and $20 \%$. The spectra recorded at $0 \%$ and $20 \%$ TFE are shown in bold. 

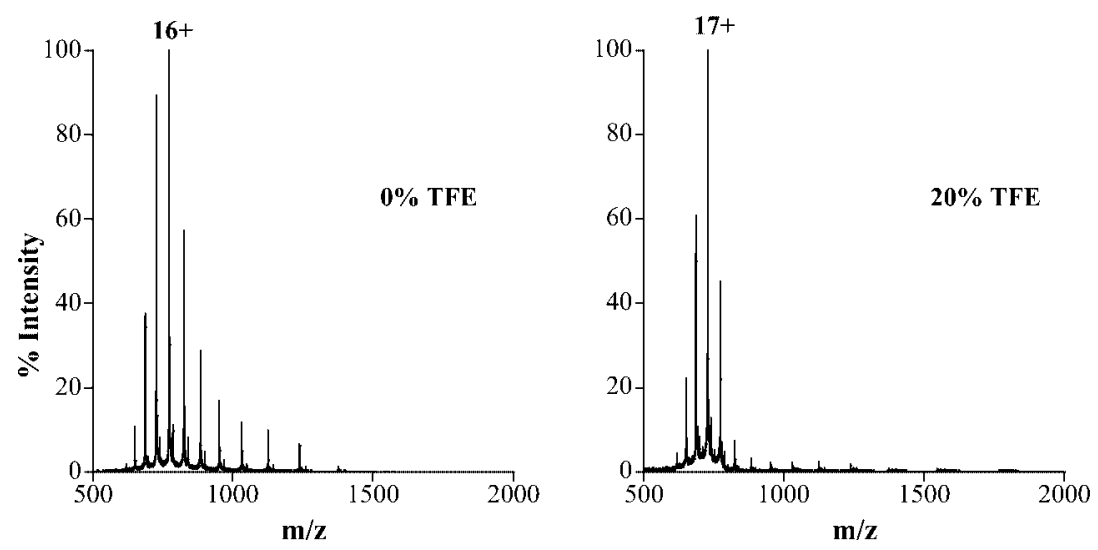

Fig. 2. Nano-ESI-MS spectra of $5 \mu \mathrm{M}$ cyt $c$ pH 2.2 at $0 \%$ and 20\% TFE. Peaks are labeled by the corresponding charge state.

analogous data on helix formation in the H-peptide. It should be underscored that CSDs in ESI-MS are largely determined by charges carried by amino-acid side chains. It is, therefore, not surprising that this technique be neutral towards hydrogen-bond formation between backbone atoms.

However, the spectra reported in Fig. 2 reveal minor changes in the CSD of unfolded cyt $c$ induced by TFE addition. The protein envelope is slightly narrowed and shifted towards lower $\mathrm{m} / \mathrm{z}$ values at $20 \%$ TFE. Similar results were obtained with the H-peptide [13]. The shift of the CSD can be interpreted as a consequence of maximal exposure of amino-acid side chains in the $\alpha$-helix. In monomeric chains, tertiary contacts can be favored more by random-coil than by helical conformations, under particular solvent conditions. The effect depicted in Fig. 2 could be due to minor residual protection of side chains towards proton transfer reactions in the denatured state. Extensive helix formation induced by TFE seems to minimize intramolecular interactions between side chains in unfolded cyt $c$, giving rise to more highly charged states in ESI-MS.

Another minor but reproducible effect of TFE on the ESI-MS spectra of denatured cyt $c$ is reducing the broadness of the CSD (Fig. 2). Two factors are supposed to contribute to the bell-shaped protein envelopes observed by ESI-MS under denaturing conditions. Statistical fluctuations in the extent of the proton transfer reactions taking place during the electrospray process, and the conformational dynamics of the polypeptidic chain $[8,18]$. The latter factor can result in partial and heterogeneous protection of ionizable groups in the denatured state. By reducing the conformational freedom of the unfolded protein, TFE offers the possibility to evaluate the specific contributions of these two effects. It cannot be ruled out $a$ priori that TFE also affects the efficiency of proton transfer reactions in the ESI droplets. However, the existence of a plateau (around 5-10\% TFE content) in the TFE effect on ESI-MS results [13] hints to a role of conformational changes in the polypeptidic chain, more than to solvent effects on the ESI process. The residual charge-state heterogeneity observed in the highly helical denatured state (Fig. 2) is most likely ascribable to fluctuations in the efficiency of the ionization process.

The addition of TFE to the acid-denatured protein does not induce appearance of CSDs at higher $\mathrm{m} / \mathrm{z}$ values, as typically observed upon protein folding. This result indicates that TFE-induced helix formation in acid-denatured cyt $c$ is not associated with stabilization of the native folded structure. The same was observed at intermediate TFE concentrations (2.5-20\%). This conclusion finds further support in the results obtained with TFE at $\mathrm{pH} \sim 3$ described below. 


\subsection{Optimization of the experimental conditions}

We carried out a systematic investigation of the influence of the instrumental parameters on protein spectra obtained by nano-ESI-MS. For this purpose, we chose as a probe the marginally stable system of cyt $c$ at $\mathrm{pH} 2.7$ (1\% acetic acid). In such a sample, the folded and unfolded states are similarly populated, and perturbation of the folding equilibrium can be readily detected by changes in the relative abundance of the two components. We found that one of the most critical parameters that need to be accurately controlled for the analysis of folding equilibria by nano-ESI-MS is the flow rate of the curtain gas (Fig. 3). Stable spectral features are observed only in the small range between 0.5 and $0.7 \mathrm{l} / \mathrm{min}$. Higher flow rates result in a progressively higher proportion of unfolded protein, which eventually reaches $100 \%$ at a gas flow rate of $1 \mathrm{l} / \mathrm{min}$. Protein unfolding is most probably induced, in this case, by the heat produced during collision of sample droplets with the nitrogen gas. At gas flow rates below $0.5 \mathrm{l} / \mathrm{min}$, no stable spray was obtained. For the measurements reported in Fig. 3, the same sample was sprayed at progressively higher gas flow rates and then brought back to the initial condition. Reversal to the original spectrum indicates stability of the sample during the whole experiment. In all the conditions explored here, protein unfolding in vacuo, i.e., downstream the ESI process, is irrelevant, since it would not alter the observed charge state of protein ions [19].

Analogous results were obtained by varying the nozzle potential and the spray-tip potential (data not shown), although these factors appear to be less effective than the curtain gas flow rate inducing protein unfolding. The results obtained by varying these parameters indicated that a nozzle-to-skimmer potential (cone voltage) between 13 and $73 \mathrm{~V}$ and a spray-tip potential between 1500 and $1700 \mathrm{~V}$ are compatible with stable spectral features. The cone voltage can be used to induce fragmentation in ESI-MS, controlling the energy of the collisions of ions or small droplets with residual gas molecules in the proximal region of the mass analyzer. We found that the cone voltage can have opposite effects (i.e., increase or decrease the apparent fraction of folded protein), depending on the value of the other parameters. Different mechanisms can contribute to these complex effects, such as protein unfolding, fragmentation and deprotonation $[16,20]$. Further experiments are required, in order to evaluate the different contributions of these mechanisms. Protein unfolding could also be induced adjusting the nozzle temperature above $120^{\circ} \mathrm{C}$ (data not shown). The spray-tip potential was found to have the least influence on conformational stability during the electrospray process and, rather, to affect dramatically the stability of the electrospray. All together, these experiments indicate that it is possible to define conditions for nano-ESI-MS of protein solutions under which the instrumental parameters do not limit protein stability during the electrospray process.

\subsection{Detection of folding intermediates}

The experiments described here, together with other evidence reported in the literature [18,21-23], indicate that compactness is the feature of protein structures that ESI-MS monitors with high sensitivity. Interestingly, compactness is also the property discriminating different stages of the folding process. On the basis of these observations, ESI-MS stands out as a very promising method for detection of folding intermediates that has the potential to effectively discriminate these forms from both the folded and the unfolded states. This has been proved to be the case for the well characterized proteins myoglobin [23-25] and cyt $c$ [26]. The work recently carried out by our group focuses on equilibrium folding intermediates of cyt $c$ under oxidizing conditions [26]. This protein undergoes a highly cooperative folding transition [27] between $\mathrm{pH} 3$ and $\mathrm{pH} 2$. Conditions known to stabilize the cyt $c$ molten-globule state 

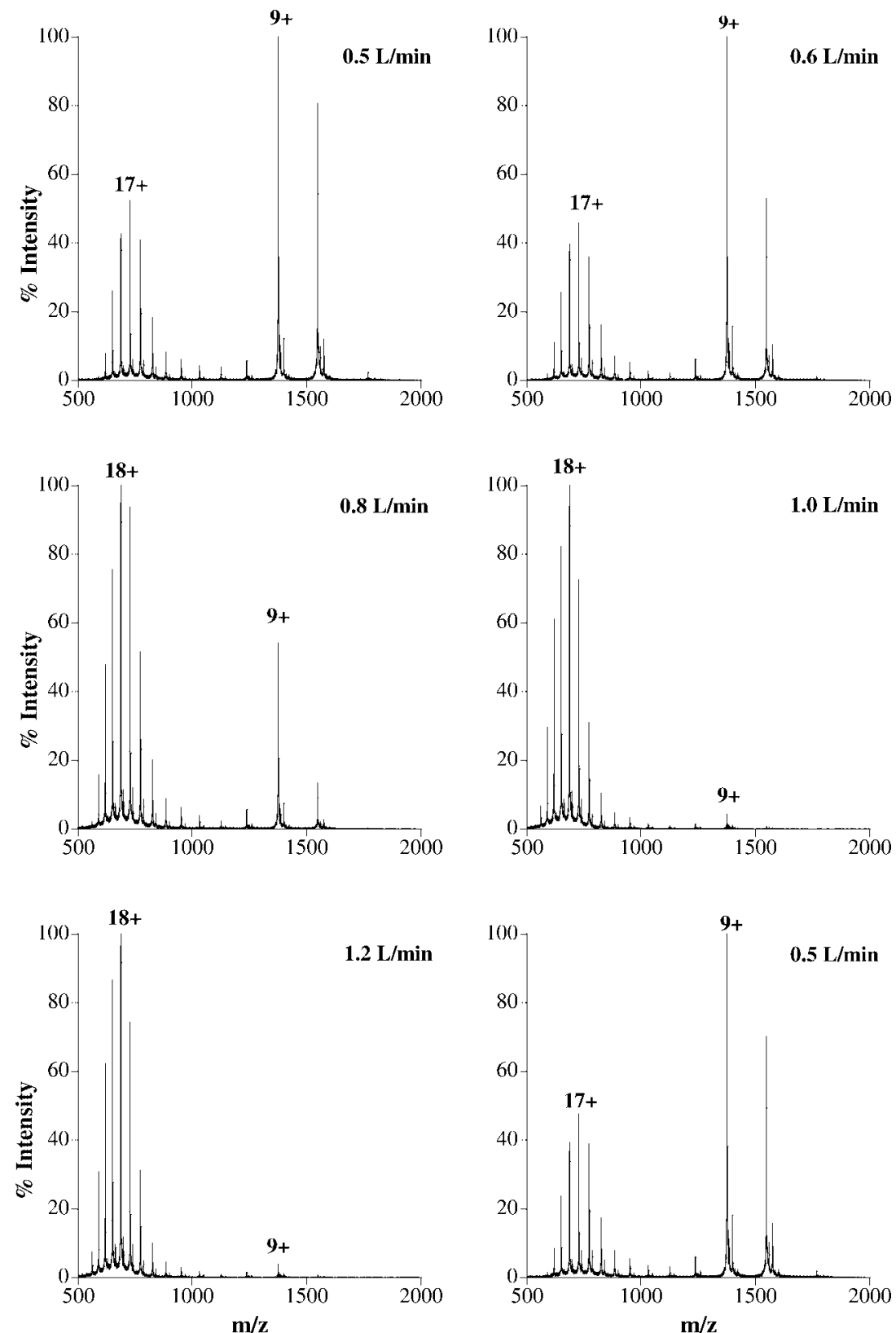

Fig. 3. Nano-ESI-MS spectra of $5 \mu \mathrm{M}$ cyt $c$ in water-acetate $\mathrm{pH} 2.7$ at varying gas flow rate (as indicated in each panel). Peaks are labeled by the corresponding charge state.

were found to give rise to intermediate CSDs in ESI-MS, mapping between those of the folded and the unfolded protein $[21,26]$. These conditions are $10 \%$ glycerol at $\mathrm{pH} \sim 2$, and $25 \%$ methanol at $\mathrm{pH} \sim 3$. Indeed, the folding equilibrium can be shifted towards the molten-globule state by the addition of stabilizing agents to the denatured protein, or by the addition of denaturing agents to the native protein. Small amounts of these intermediate forms could be detected, even in the absence of additives, around the mid point of the acid-induced unfolding transition (i.e., $\mathrm{pH}$ 2.4-2.5). This result provides direct evidence for 

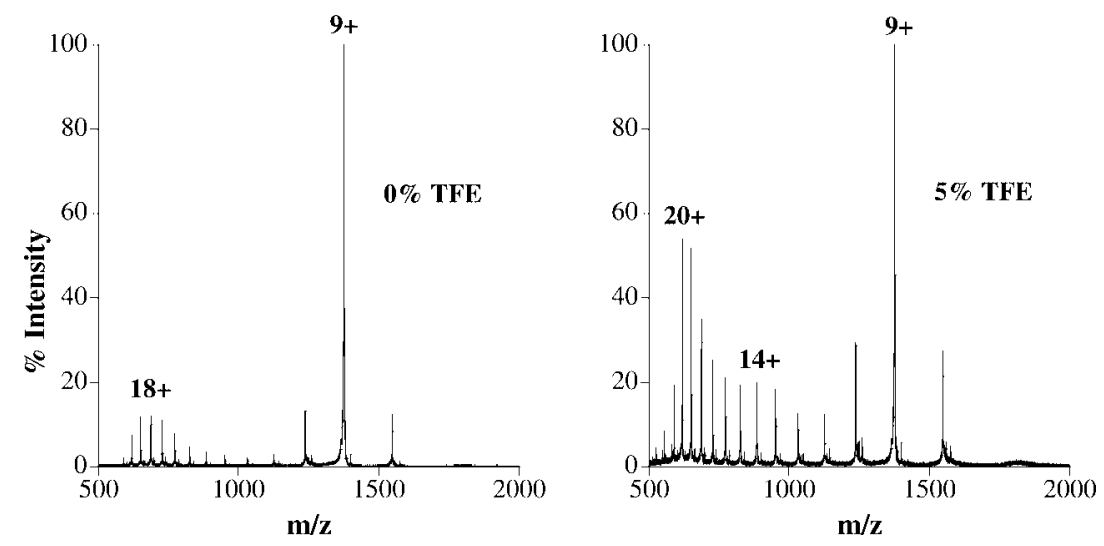

Fig. 4. Nano-ESI-MS spectra of $5 \mu \mathrm{M}$ cyt $c$ pH 2.8 at $0 \%$ and 5\% TFE. Peaks are labeled by the corresponding charge state.

involvement of equilibrium folding intermediates in the conformational transitions of cyt $c$ at low $\mathrm{pH}$, and is in agreement with evidences based on absorption spectrophotometry [27]. These studies [26] also indicated that at least two distinct partially folded forms $\left(I_{\mathrm{A}}\right.$ and $\left.I_{\mathrm{B}}\right)$ characterize the equilibrium folding intermediates of cyt $c$.

After establishing that we could monitor formation of known equilibrium folding intermediates by ESI-MS, this method was employed to explore novel solvent conditions for the stabilization of the cyt $c$ molten globule. Low concentrations of TFE at $\mathrm{pH} \sim 3$ were found to induce formation of a cyt $c$ partially folded form [26]. As indicated by the spectra in Fig. 4, the protein in water/acetate at pH 2.8 is mostly in its native state ( $\sim 65 \%$ folded). The addition of $5 \%$ TFE to this solution results in protein unfolding ( $\sim 34 \%$ folded) with accumulation of a partially folded form $(\sim 21 \%)$ similar to $I_{\mathrm{B}}$. This result indicates that it is possible to obtain a TFE-induced cyt $c$ molten globule via destabilization of native tertiary structure and not, as previously suggested [28], via stabilization of secondary structure in the acid-denatured protein.

\section{Discussion}

Nano-ESI-MS is a powerful method for the analysis of heterogeneous protein samples, characterized by high sensitivity and high throughput. The work described here discusses its applicability to the study of protein conformational states in solution. Comparison of CD and ESI-MS results on the effect of TFE on model compounds indicates that ESI-MS is not directly sensitive to the content of secondary structure in polypeptidic samples. These results corroborate the previously suggested specificity of this method for monitoring changes in protein tertiary structure. It is shown that the coil-to-helix transition per se does not affect the ESI-MS spectra of peptides and proteins. Thus, ESI-MS offers complementary information to that usually obtained by $\mathrm{CD}$ or hydrogen exchange. While this conclusion can be rationalized on the basis of involvement of main-chain atoms in secondary structure formation, some exceptions to this rule are possible. For instance, involvement of ionizable side chains in helix capping, or in the substitution of main-chain atoms in the hydrogen-bond network stabilizing secondary structure $[29,30]$, could influence the charge states observed in ESI-MS.

The neutrality of ESI-MS towards local main-chain conformation can be exploited in order to investigate the effect of secondary structure formation on protein tertiary and quaternary structure. Data 
presented here indicate that helix stabilization by TFE in acid-denatured cyt $c$ at $\mathrm{pH} 2.2$ does not induce tertiary structure formation. This result is in contradiction with the hypothesis [28] of stabilization of the cyt $c$ molten globule by low concentrations of TFE at $\mathrm{pH} 2.2$, and suggests that the more compact conformation induced by the additive under these conditions be due only to a higher helix content and not to more extensive tertiary contacts, as compared to the unfolded state. Peptide models for the complex between cyt $c$ terminal helices and the heme indicated that tertiary structure formation induces coil-tohelix transition in these protein fragments. The synergic role of secondary and tertiary structure in the stabilization of native protein conformations suggests that stabilizing helical structure would stabilize globular conformations. However, this does not seem to be the case when helix stabilization is achieved by TFE addition at low $\mathrm{pH}$. Under these conditions, the net effect of the additive (even at low concentrations such as $2.5-7.5 \%$ ) is to destabilize tertiary structure, as also indicated by TFE-induced unfolding at $\mathrm{pH}$ 2.8. Most probably, the reduced solvophobic effect in the presence of TFE, and/or interactions of the cosolvent with the polypeptidic chain [14], become the predominant factors affecting tertiary structure stability at low $\mathrm{pH}$. This conclusion is consistent with the view of an already destabilized tertiary structure of cyt $c$ at low $\mathrm{pH}$, due to unfavorable side-chain ionization [27]. Formation of non-native secondary structure might also play a role. However, the observation [14] that cyt $c$ folds to its native conformation, with almost unperturbed kinetics, at pH 6 in the presence of low TFE concentrations seems to rule out this hypothesis.

Experiments described here investigate the influence of the experimental conditions on nano-ESI-MS analysis of marginally stable protein structures. Several instrumental parameters can affect the apparent fraction of unfolded protein in the spectra of cyt $c$ at $\mathrm{pH}$ 2.7. Each of them can be set to limit conformational stability in the protein sample during the ESI process. Nevertheless, a range of values can be found in which stable spectral features are observed. Therefore, highly controlled operational conditions allow to obtain reliable information about the different conformational states populated in the protein sample. This conclusion is further supported by the good agreement that has been achieved between ESI-MS and other spectroscopical methods identifying solvent conditions for protein denaturation [23,26,31].

We show that collisions with the nitrogen gas in the nano-ESI interface can induce protein unfolding in the ESI droplets. Collisions with nitrogen or argon gas could also be used to induce unfolding of myoglobin ions in the gas phase [32]. An influence of drying-gas on protein CSDs in ESI-MS has been already observed. The observation of higher charge states at higher gas flow rates has been related to the ionization mechanism [20,33]. In this view, the time point at which a multiply charged ion is transferred to the gas phase, during the evolution of ESI droplets, defines its final charge state. Higher flow rates would result in faster droplet evaporation and, therefore, in a higher average charge density on the droplet surface at the moment of production of gas-phase ions. The results reported here indicate that higher gas flow rates can result in higher average charge states also due to protein unfolding. This conclusion, clearly supported by the interconversion between two distinct CSDs, is not linked to a particular model for ion formation (ion-evaporation [34] versus charged-residue [35] model).

ESI-MS, and nano-spray techniques in particular, have been successfully employed for detection of folding intermediates. These studies showed that these methods can increase the resolution at which we describe protein conformational transitions. Two distinct partially folded forms were found to populate the cyt $c$ molten-globule state [26]. Similar intermediates were detected at $\mathrm{pH} \sim 2$ in the presence of glycerol, $\mathrm{pH} \sim 3$ in the presence of methanol or TFE, and at $\mathrm{pH} 2.4-2.5$ in the absence of additives. The consistence of results from different solvent and experimental conditions strongly suggest that this method monitors protein-specific structural features. The high sensitivity of ESI-MS to protein tertiary 
structure not only discriminates folded and unfolded states, but also allows monitoring minor conformational changes, like the transition from molten globule to native state or structural compression associated to protein stabilization $[21,26]$.

MS methods offer a particular advantage for folding studies: not averaging the output signal over the molecular ensemble. Rather, they provide distinct information about the different populations present in the protein sample, free from the background of the other components. This property is a direct consequence of the ion-sorting mechanism inherent to any MS measurement. This feature makes ESI-MS a powerful method for detection of poorly populated conformational states of proteins in solution. The unique advantages of MS analyzing heterogeneous samples can now be exploited for the characterization of dynamic equilibria involving different conformational states.

\section{Materials and methods}

Horse heart type VI cyt $c$ and TFE were purchased from Sigma, and methanol from Merck. Protein samples were prepared in water/acetic acid mixtures without further purification. The $\mathrm{pH}$ was adjusted to the desired value with diluted acetic acid (a pH value of 2.4 corresponds to an acetic acid content of $\sim 4 \%$ ). CD spectra were recorded on a Jasco J-810 instrument in a $0.1 \mathrm{~mm}$ cuvette, at $20^{\circ} \mathrm{C}$.

Mass spectra were recorded on a Mariner time-of-flight, ESI mass spectrometer (Perkin Elmer) in the positive-ion mode using a nano-electrospray sample interface. When not differently indicated, the spray tip potential was set at $1600 \mathrm{~V}$, the nozzle potential at $20 \mathrm{~V}$, the skimmer potential at $7 \mathrm{~V}$, the nozzle temperature at $80^{\circ} \mathrm{C}$, and the nitrogen curtain gas flow rate to $0.6 \mathrm{l} / \mathrm{min}$. The instrumental parameters were optimized by tuning the instrument on the $9+$ peak of cyt $c$ at $\mathrm{pH} 3$. Samples were sprayed at $\sim 25^{\circ} \mathrm{C}$. The capillaries were purchased from Protana (inner diameter of $0.69 \mathrm{~mm}$ and "medium" length of the outermost tip). The software for data recording (Mariner ${ }^{\mathrm{TM}}$ Workstation version 4.0 ) and data processing (Data Explorer ${ }^{\mathrm{TM}}$ version 3.2) was supplied by Applied Biosystems. Spectra were recorded with an integration time of $3 \mathrm{~s}$ and averaged over at least $30 \mathrm{~s}$.

\section{Acknowledgements}

We thank Prof. Wolfgang Buchberger for helpful discussions. This work was funded by the grants H147-CHE (Charlotte-Bühler fellowship for R.G.) and P13906-CHE of the Austrian Science Fund (FWF).

\section{References}

[1] J.L. Apuy, Z.-Y. Park, P.D. Swartz, L.J. Dangott, D.H. Russell and T.O. Baldwin, Pulsed-alkylation mass spectrometry for the study of protein folding and dynamics: development and application to the study of a folding/unfolding intermediate of bacterial luciferase, Biochemistry 40 (2001), 15 153-15 163.

[2] V. Katta and B.T. Chait, Conformational changes in proteins probed by hydrogen-exchange electrospray-ionization mass spectrometry, Rapid Commun. Mass Spectrom. 5 (1991), 214-217.

[3] S.K. Chowdhurry, V. Katta and B.T. Chait, Probing conformational changes in proteins by mass spectrometry, J. Am. Chem. Soc. 112 (1990), 9012-9013.

[4] G. Siuzdak, The emergence of mass spectrometry in biochemical research, Proc. Natl. Acad. Sci. USA 91 (1994), 11290 11297.

[5] R. Grandori, Electrospray-ionization mass spectrometry for protein conformational studies, Curr. Org. Chem. (2002) (in press). 
[6] F. Halgand and O. Laprevote, Mean charge state and charge state distribution of proteins as structural probes. An electrospray ionisation mass spectrometry study of lysozyme and ribonuclease A, Eur. J. Mass Spectrom. 7 (2001), 433-439.

[7] C. Meunier, M. Jamin and E. De Pauw, On the origin of the abundance distribution of apomyoglobin multiply charged ions in electrospray mass spectrometry, Rapid Commun. Mass Spectrom. 12 (1998), 239-245.

[8] R. Grandori, On the origin of the conformation dependence of protein charge-state distributions in electrospray-ionization mass spectrometry, J. Mass Spectrom. (2002) (in press).

[9] V. Katta and B.T. Chait, Observation of the heme-globin complex in native myoglobin by electrospray-ionization mass spectrometry, J. Am. Chem. Soc. 113 (1991), 8534-8535.

[10] T.D. Veenstra, K.L. Johnson, A.J. Tomlinson, R. Kumar and S. Naylor, Correlation of fluorescence and circular dichroism spectroscopy with electrospray ionization mass spectrometry in the determination of tertiary conformational changes in calcium-binding proteins, Rapid Commun. Mass Spectrom. 12 (1998), 613-619.

[11] X.M. Pan, X.R. Sheng and J.M. Zhou, Probing subtle acid-induced conformational changes of ribonuclease A by electrospray mass spectrometry, FEBS Lett. 402 (1997), 25-27.

[12] L. Konermann and D.J. Douglas, Acid-induced unfolding of cytochrome $c$ at different methanol concentrations: electrospray ionization mass spectrometry specifically monitors changes in the tertiary structure, Biochemistry 36 (1997), $12296-12302$.

[13] R. Grandori, I. Matecko and N. Müller, Uncoupled analysis of secondary and tertiary protein structure by circular dichroism and electrospray ionization mass spectrometry, J. Mass Spectrom. 37 (2001), 191-196.

[14] D. Hamada, F. Chiti, J.I. Guijarro, M. Kataoka, N. Taddei and C.M. Dobson, Evidence concerning rate-limiting steps in protein folding from the effects of trifluoroethanol, Nature Struct. Biol. 7 (2000), 58-61.

[15] A. Cammers-Goodwin, T.J. Allen, S.L. Oslick, K.F. McClure, J.H. Lee and D.S. Kemp, Mechanism of stabilization of helical conformations of polypeptides by water containing trifluoroethanol, J. Am. Chem. Soc. 118 (1996), 3082-3090.

[16] T.A. Fligge, K. Bruns and M. Przybylski, Analytical development of electrospray and nanoelectrospray mass spectrometry in combination with liquid chromatography for the characterization of proteins, J. Chromatogr. B Biomed. Sci. Appl. 706 (1998), 91-100.

[17] M.S. Wilm and M. Mann, Electrospray and Taylor-cone theory, Dole's beam of macromolecules at last?, Int. J. Mass Spectrom. Ion Proc. 136 (1994), 167-180.

[18] D.A. Simmons and L. Konermann, Characterization of transient protein folding intermediates during myoglobin reconstitution by time-resolved electrospray mass spectrometry with on-line isotopic pulse labeling, Biochemistry 41 (2002), 1906-1914.

[19] P. Kebarle and Y. Ho. Titla, in: Electrospray Ionization Mass Spectrometry, R.B. Cole, ed., John Wiley \& Sons, New York, 1997, pp. 3-63.

[20] G. Wang and R.B. Cole. Titla, in: Electrospray Ionization Mass Spectrometry, R.B. Cole, ed., John Wiley \& Sons, New York, 1997, pp. 137-174.

[21] R. Grandori, I. Matecko, P. Mayr and N. Müller, Probing protein stabilization by glycerol using electrospray mass spectrometry, J. Mass Spectrom. 36 (2001), 918-922.

[22] A. Miranker, C.V. Robinson, S.E. Radford and C.M. Dobson, Investigation of protein folding by mass spectrometry, FASEB J. 10 (1996), 93-101.

[23] A. Dobo and I.A. Kaltashov, Detection of multiple protein conformational ensembles in solution via deconvolution of charge-state distributions in ESI MS, Anal. Chem. 73 (2001), 4763-4773.

[24] L. Konermann, F.I. Rosell, A.G. Mauk and D.J. Douglas, Acid-induced denaturation of myoglobin studied by timeresolved electrospray ionization mass spectrometry, Biochemistry 36 (1997), 6448-6454.

[25] K.R. Babu and D.J. Douglas, Methanol-induced conformations of myoglobin at pH 4.0, Biochemistry 39 (2000), 14702 14710.

[26] R. Grandori, Detecting equilibrium cytochrome $c$ folding intermediates by electrospray ionization mass spectrometry: two partially folded forms populate the molten-globule state, Protein Sci. 11 (2002), 453-458.

[27] Y. Goto, Y. Hagihara, D. Hamada, M. Hoshino and I. Nishii, Acid-induced unfolding and refolding transitions of cytochrome c: a three-state mechanism in $\mathrm{H}_{2} \mathrm{O}$ and $\mathrm{D}_{2} \mathrm{O}$, Biochemistry 32 (1993), 11 878-11 885.

[28] T. Konno, J. Iwashita and K. Nagayama, Fluorinated alcohol, the third group of cosolvents that stabilize the molten-globule state relative to a highly denatured state of cytochrome c, Protein Sci. 9 (2000), 564-569.

[29] D. Bordo and P. Argos, The role of side-chain hydrogen bonds in the formation and stabilization of secondary structure in soluble proteins, J. Mol. Biol. 243 (1994), 504-519.

[30] D. Gandini, L. Gogioso, M. Bolognesi and D. Bordo, Patterns in ionizable side chain interactions in protein structures, Proteins 24 (1996), 439-449.

[31] L. Konermann, B.A. Collings and D.J. Douglas, Cytochrome $c$ folding kinetics studied by time-resolved electrospray ionization mass spectrometry, Biochemistry 36 (1997), 5554-5559.

[32] Y. Konishi and R. Feng, Conformational stability of heme proteins in vacuo, Biochemistry 33 (1994), $9706-9711$. 
[33] J.B. Fenn, Ion formation from charged dropplets: roles of geometry, energy, and time, J. Am. Soc. Mass Spectrom. 4 (1993), 524-535.

[34] J.V. Iribarne and B.A. Thomson, On the evaporation of small ions from charged droplets, J. Chem. Phys. 64 (1976), $2287-2294$.

[35] M. Dole, L.L. Mack, R.L. Hines, R.C. Mobley, L.D. Ferguson and M.B. Alice, Molecular beams of macroions, J. Chem. Phys. 49 (1968), 2240-2249. 


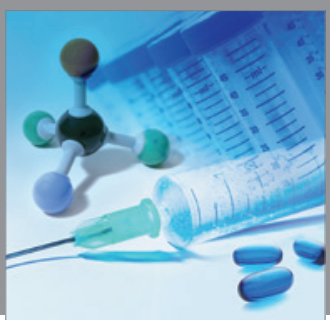

International Journal of

Medicinal Chemistry

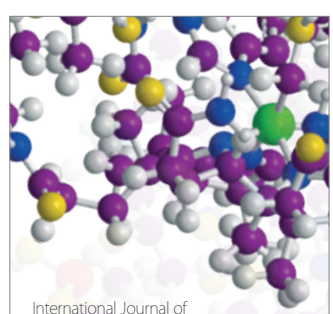

Carbohydrate Chemistry

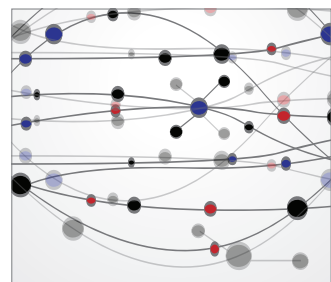

The Scientific World Journal
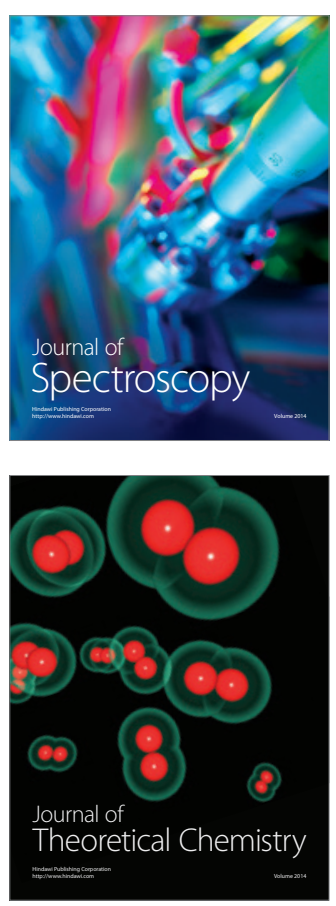
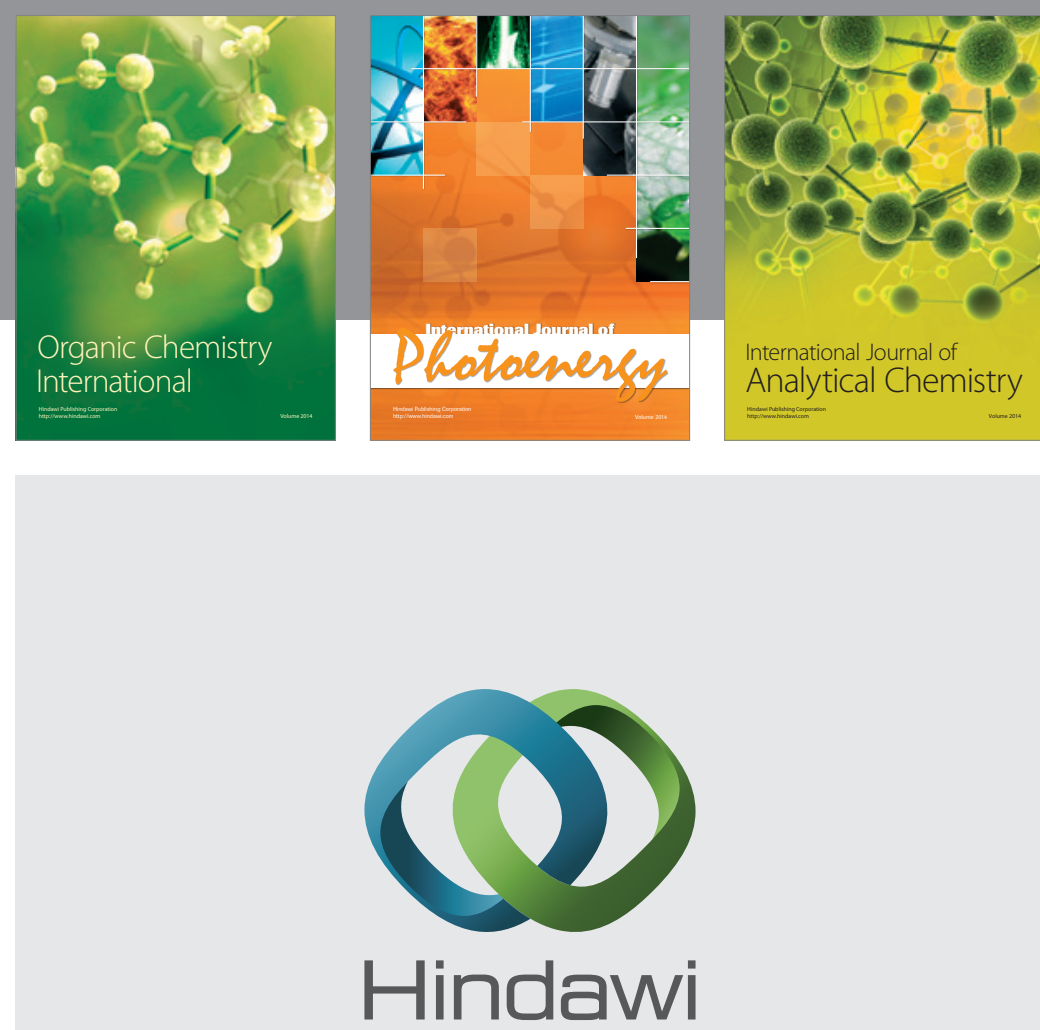

Submit your manuscripts at

http://www.hindawi.com
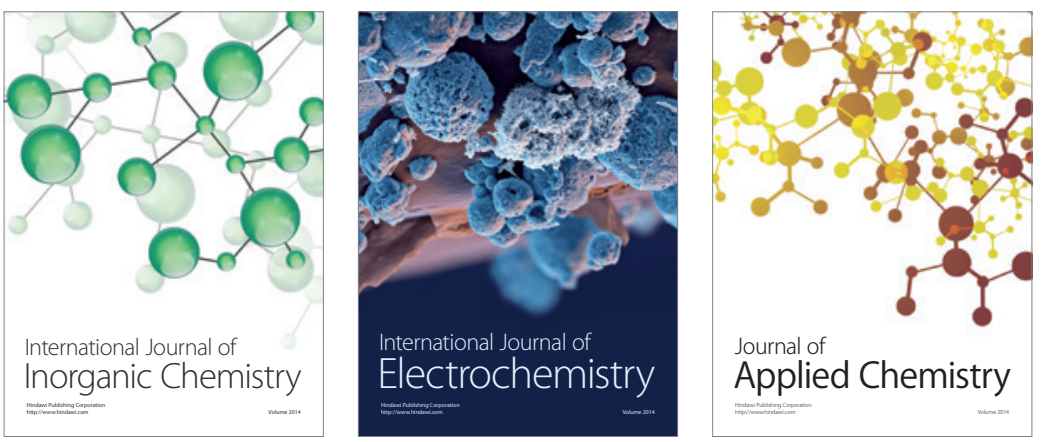

Journal of

Applied Chemistry
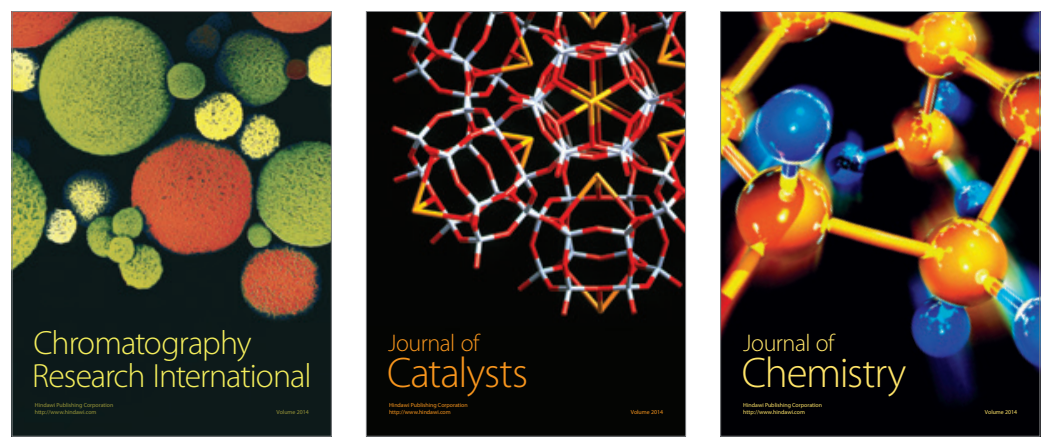
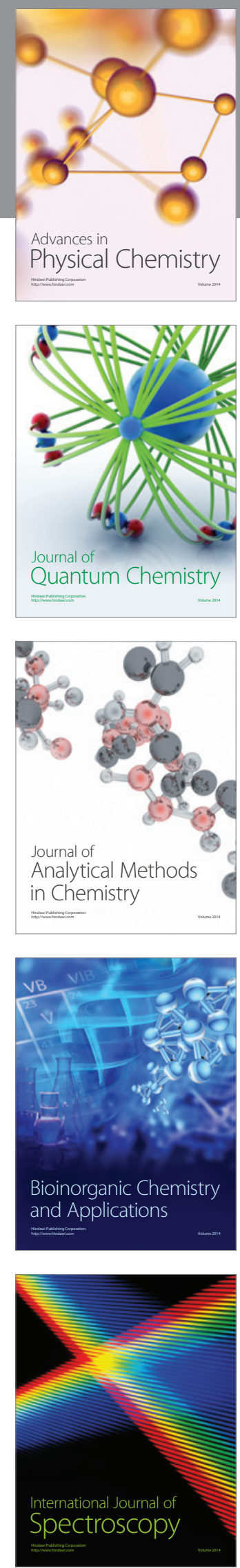